\title{
THE PROGNOSIS OF SYPHILIS IN THE LIGHT OF MODERN METHODS OF TREATMENT *
}

By L. W. HARRISON, D.S.O., M.B., F.R.C.P. (Ed.)

As I understand it, the question on which you have invited me to open this discussion is the effect of a syphilitic infection on the expectation of life when the infection is fought by modern methods. Before attempting to answer such a question it is necessary to be clear on the meaning of modern methods, since the term is applied to a wide variety of treatments which have in common only the use of arsenobenzol, under the guidance of serum tests. The mildest consists of a course of six or eight injections of "9I4" and no more unless the Wassermann reaction remains, or, later, becomes positive. Next to this "Wait and See" method, as I would call it, is a fairly common one in which the serum is rendered negative by arsenobenzol and mercury or bismuth, and the patient is then treated with mercury for two years. The principle of this method seems to be that the negative serum reaction may not indicate cure, but that this is assured in any case by two years of mercurial treatment because this was the amount of treatment considered to be sufficient by the late Sir Jonathan Hutchinson. If I am to state my views on these methods, I would refer you to articles on the prognosis of syphilis in the preWassermann era and say that, by such treatment, the outlook may have been made slightly better than in the pre-Wassermann era by reason of the fact that in many cases the serum tests would indicate a resumption of treatment which would otherwise have remained in abeyance until a clinical relapse occurred. Certainly it is better for the patient that treatment should be resumed as early as possible on any sign that the disease has not been eradicated, but all our experience goes to show that,

* Based upon a paper read before the Assurance Medical Society on March 2nd, I927.

v.D: 


\section{BRITISH JOURNAL OF VENEREAL DISEASES}

if we allow a relapse to occur, it is a much more difficult task to secure complete sterilisation. Further, the serological relapse often passes unnoticed and the clinical relapse is often in a form which has gained for syphilis the reputation of being one of the great killing diseases. That relapses are frequent under the short course of arsenobenzol and mercury followed by two years' mercurial treatment method of management is shown by the results of blood tests made by Glynn, Roberts and Bigland (Medical Research Council, Special Report Series No. I07). These workers found in 228 cases treated within three months of infection with "9I4" and mercury until the Wassermann reaction was negative and then with mercury, that thirty-nine relapsed within six months of the arsenobenzol being stopped, whilst, of course, the patients were under mercurial treatment. Of the remaining I89 in this class, I20 continued under mercurial treatment for seven to twelve months, and blood tests of these showed twenty-three relapses.

Glynn and colleagues, in fact, found a larger percentage of relapses in those whose treatment (commenced within three months of infection) was continued with mercury after the attainment of a negative Wassermann reaction than in those who received no such continuation treatment. These were defaulters who turned up at various times later and were tested. Out of I23 who turned up again within six months, eight were found to have relapsed serologically, and in 135 who returned seven to twelve months after the arsenobenzol course, eighteen relapses were discovered.

These results were not very surprising to me as my own tests, which I will discuss later, had taught me that purely mercurial treatment does little or nothing towards the eradication of syphilis. The work of Glynn and colleagues, in fact, confirms my opinion that a short course of arsenobenzol and mercury cures only a small proportion of cases, and that continuation treatment with mercurial treatment alone is useless from the point of view of eradicating the disease in those not cured by the preceding arsenobenzol and mercurial treatment. I think it better, therefore, to base my remarks on the prognosis of syphilis on results obtained when the disease has been treated on lines more strenuous than those I have mentioned. The principles of this method of management require that 


\section{PROGNOSIS OF SYPHILIS}

treatment shall be continued by injections of an arsenobenzol compound and of mercury, or bismuth, to the extent of not less than twenty each (totalling I3 to I4 grm. "9I4"), beyond the stage when the serum tests first give negative results, and that it shall not be stopped even then unless the cerebro-spinal fluid is also negative. The purely specific treatment in such a scheme of management is naturally supplemented, as circumstances may dictate, by iodides and by the injections of drugs which seem likely to make the metallic treatment more tolerable, such as sodium thiosulphate : or to improve its effect, such as protein-shock-producing remedies and malarial inoculation. Further, I must postulate that, after suspension of treatment, the patient is observed for at least two years, not only clinically, but by tests of the serum and spinal fluid.

The principles which I have just outlined are followed in many treatment centres in Great Britain, including my own, and in such as the Mayo Clinic and that of the Johns Hopkins Hospital, besides many on the Continent. When syphilis is treated on these lines the outlook depends on the stage of the disease at which it commenced, and it is necessary to discuss the question from this point of view, summing up the prospects in each of a number of representative types of case. I will deal first with the most favourable, viz., the case of the person who is treated from the earliest days of the infection before the serum reactions have become positive. The evidence is strongly in favour of the probability that in the great majority of such cases the disease can be eradicated entirely. I am aware that some clinicians hold that syphilis can never be eradicated, and that the only safety for the syphilitic lies in treatment every year for the rest of his life. I am quite ready to believe that, if such a pessimistic view is founded on experience of cases which have been treated with mercury alone, or mainly with mercury, it may be justified, but it is contrary to evidence, both experimental and clinical, which shows that, when arsenobenzol is employed, complete sterilisation can be effected. I should like to cite first the experimental evidence. Animals, such as rabbits, can be infected with syphilis, and the disease becomes generalised much as in men. It is reasonable to suppose, therefore, that the behaviour of infected animals under treatment is an 


\section{BRITISH JOURNAL OF VENEREAL DISEASES}

index of the happenings in man. Kolle (Deutsch. med. Woch., I922, No. 39, p. I30I ; I924, No. 37, p. I234) showed that sterilisation of infected rabbits could be effected by arsenobenzol treatment in 85 per cent. of cases when the treatment was instituted in the first thirty days (forty-five rabbits), proof of sterilisation being ability to re-infect the treated animals. When the treatment was delayed until the ninetieth day after the first infection sterilisation, as judged by ability to re-infect, was effected in only two out of twelve rabbits. In his later paper (I924) Kolle showed that, when treatment was instituted later than the ninetieth day, re-infection always failed, even though the treatment was for six months with salvarsan, mercury and bismuth. It seems possible that failure to re-infect 90 per cent. of rabbits whose treatment commenced so long as ninety days after infection may not have been due to failure to sterilise in the first place, but to the development in the rabbits of a more lasting immunity. This at least would seem to be the inference to be drawn from the experiments of Chesney and Kemp (Jour. Exper. Med., I926, vol. 44, p. 589). These workers tested for eradication of the original infection by inoculation of other animals with material from the lymph glands of the treated animals, lymph glands having been shown by Pearce and Brown (Jour. Exper. Med., January, I922) and others to be the most likely harbourers of $S p$. pallida in uncured syphilis of rabbits. Chesney and Kemp found in twenty-three rabbits treated with arsenobenzol that the longer the infection has lasted when sterilisation is effected, the less likely the re-infection to succeed. So far they agree with Kolle, but they do not accept Kolle's view that failure to re-infect indicates that treatment had failed since they proved success of their treatment by failing to infect other animals by inoculating them with emulsion of the lymph nodes of their treated animals. It is not quite clear from Kolle's later articles whether or not he employed the lymph node transfer method of testing the success of his treatment, as he makes only a general statement that, in latent syphilis of rabbits as also in latent syphilis of men, inoculation with material from their organs into other animals results in infection of these. I interpret his remarks on this point as indicating that he did not apply this test, and Chesney and Kemp say definitely that Kolle has regarded failure: 


\section{PROGNOSIS OF SYPHILIS}

to re-infect " without, so far as we have been able to ascertain, carrying out any observations to determine whether or not the internal organs of treated syphilitic rabbits are infectious," so that they also seem to be in some doubt as to the interpretation of Kolle's remarks on this point. This small discussion may seem to be wide of one on prognosis, but it appears to me important since the behaviour of rabbits to syphilitic infection and their reaction to treatment in the early stages at any rate, seem to be very similar to those in man. Kolle, on the one hand, says that an animal cannot be sterilised (or is sterilised in only ro per cent. of cases) when treatment commences after the ninetieth day, while Chesney and Kemp, on the other hand, say that it can. If Kolle is right, and if one can argue from rabbit to man, eradication of syphilis is hopeless after about the third month. If Chesney and Kemp are right, eradication is possible when treatment is commenced long after the ninetieth day. Experience with the treatment of men seems to indicate that Chesney and Kemp's view is more likely to be correct. In any case there is complete agreement as to the curability of syphilis in the earlier stages and as to the importance of commencing treatment as early as possible.

Incidentally, other experiments by Kolle have shown that, when tested under the same conditions, both mercury and bismuth are far behind the arsenobenzol compounds in therapeutic power. Indeed, the evidence rather indicates that they do not act directly on $S p$. pallida, but indirectly by making the tissues inhospitable.

In the case of human syphilis direct refutation of the statements by pessimists that the disease is never eradicated is impossible because, of course, we cannot cut up our patients and inoculate bits of them into animals, and negative results of such a procedure would be the only absolute means of proving that the pessimists are wrong. But it seems justifiable to say that, if after such and such a course of treatment a patient has remained absolutely free from signs, both clinical and serological as well as in respect of the cerebro-spinal fluid, for a number of years, that patient has most probably been freed from infection. The existence of many such cases in the experience of various observers and the fact that there have been many instances of re-infection is, I think, good evidence that syphilis can, in favourable circumstances, be eradicated. 


\section{BRITISH JOURNAL OF VENEREAL DISEASES}

In what proportion of cases treated on good lines before the serum reactions have become positive can we expect eradication of the disease, the fact of eradication being proved by complete freedom from signs both clinical and serological for one or more years after suspension of treatment? Perhaps the most searching investigation on this point is that by Moore and Kemp (Johns Hopkins Hospital Bulletin, July, I926). These workers were satisfied with nothing short of complete absence of pathological signs not only in the blood, but also in the cerebro-spinal fluid. Their material consisted in the gross of 2,500 patients with primary or secondary syphilis treated during nine years in the Syphilis Clinic of the Johns Hopkins Hospital. Out of this number they were able to report on only forty-two patients with seronegative primary syphilis treated and observed sufficiently long to enable judgment to be formed on the results. They found that out of seventeen of these cases which received from one to eight injections of " 606 " with no mercury at all, or mercurial inunctions for less than one month after an arsenobenzol course, two became reinfected, one was free from signs for one to two years, one for two to three years, and thirteen relapsed in some form or another. In eleven cases which had received six to twelve injections of " 606 " with at least two months of mercurial inunction, there were five probable cures, viz., one re-infection, one free from relapse for one to two years later, one free for two to three years, and two free for three to four years. Six recurrences happened in this group. In seven treated with thirteen to twenty injections, with mercurial inunctions between the three courses of "606," there were six probable cures and one relapse. The longest period of observation of the cases considered to be cured was four to five years (one case), and three cases were observed for two to three years. In seven cases treated with twenty-one to forty injections of " 606 " in four or more courses there were as many as seven probable cures. Thus in fourteen cases which had received from thirteen to forty injections of " 606 ," with mercurial inunctions over a period of approximately nine months, there were thirteen from which the authors considered they had eradicated the disease. The authors conclude that, with the utmost co-operation of the patient and painstaking treatment and observation on 


\section{PROGNOSIS OF SYPHILIS}

the part of the physician, it should be possible to cure Ioo per cent. of sero-negative primary syphilis. This seems a little too optimistic, since, in my experience, there have occasionally been cases, and such have been reported in the literature, in which the patient has not responded so well to the specific treatment. One such occurred in my clinic a few weeks ago, in which a patient who had commenced treatment in the early primary stage and received, in regular courses, twenty-five injections of "9I4" and bismuth (under which his serum reaction remained negative) relapsed with a roseolar syphilide six months after completion of treatment. Such cases are rare, but their occasional occurrence makes one unable to expect quite roo per cent. of cures. In this connection it may be worth noting that an essential for success is the co-operation of the patient's tissues. Salvarsan does not act as such, but by virtue of a derivative formed by the tissues, and some patients seem unable to form this derivative in sufficient amount to do more than keep the disease in check. Kolle believes it possible that animals and men lose the power of forming the necessary derivative after they have had syphilis for some time (e.g., ninety days), but I have found some difficulty in accepting this, though, as said, I recognise that some patients' tissues are naturally inactive in this respect.

Stokes, from his experience of syphilis in the Mayo Clinic, considers that, if the patient will co-operate with the physician who treats on the principles I enunciated early in this paper, a radical cure can be obtained in 75 per cent. of primary and early secondary cases. This appears to be a cautious estimate, but it includes secondary cases, in which the outlook is not so good.

Out of numerous other series of results which have been reported, I would quote that of S. Silberstein (Archiv. $f$. Dermat. u. Syphilog., I923, vol. I43, pp. 334-364), although the treatment on which he reports does not accord with the principles which I have stated. Silberstein's results are valuable because a large proportion of the cases reported on have been observed for a number of years, though the statistics are some cases observed for only three to six months. On this point he says that those patients in the primary and secondary stage who have been treated faithfully according to the Scholtz method and have remained negative serologically for six months 


\section{BRITISH JOURNAL OF VENEREAL DISEASES}

after completion of treatment relapse later only exceptionally. Silberstein's tables do not seem quite to bear out this view, since in the tables (I. to III. and VII. to IX.) are thirty-three relapses, of which sixteen were observed more than six months after suspension of treatment, and of the sixteen there were six observed more than a year later ; in nine of the sixteen, however, no test had been carried out between three months after treatment and the detection of the relapse. He adds that in no case which has been passed as cured in the Königsberg clinic has an examination of the cerebro-spinal fluid at a later date afforded other than a negative result. The system of treatment on which the results are based differs radically from that usually followed. In an average case the patient receives in two days three to four injections of original " 606," the second injection being given four hours after the first and one or two being given on the second day, the total dose divided up in this way being 0.85 to I grm. Two or three days later the injections are repeated, and the patient is put for four. weeks on an energetic cure with mercury, by inunctions and injections. At the end of this time another series of four " 606 " is given, this time totalling 0.65 to $0.8 \mathrm{grm}$. If the serum is now negative, the course is completed with two to three weeks of mercury, but if it is positive, a three weeks' course of mercury is followed by a third series of " 606 ," totalling $0 \cdot 6$, and this again by two weeks of mercury. Naturally the procedure is varied according to individuals, but in susceptible patients it is the individual dose rather than the total which is reduced, since Scholtz regards 0.6 to 0.7 of original " 606 " to be the minimum total which should be given in a two-day series.

The statistics are divided into those of the clinic and those of Scholtz's private patients since, as most syphilologists know, private patients are far more faithful and satisfactory than clinic.

In sero-negative primary syphilis forty-one private patients showed no relapses in periods of observations varying from six months (eight cases) to eleven years (two cases), the average period of subsequent observation being approximately two and half years.

In thirty clinic cases there were two relapses, both clinical. Four re-infections are recorded. Four of the 


\section{PROGNOSIS OF SYPHILIS}

cases were observed for more than five years and five for three to five years. Eight were under observation less than six months.

My own cases have not yet been analysed so carefully as those which I have quoted, but I can say that so far the relapses in cases of very early syphilis treated with a minimum of twenty injections of "9I4" and the same number of mercury or bismuth have been almost negligible.

Altogether it seems safe to say that, if treatment commences before the serum reaction becomes positive and is carried out on modern lines, a very high proportion of cures can be expected, certainly not less than 95 per cent.

The primary case with positive serum reactions and the early secondary may be considered together. It is generally agreed that, after the serum reactions have become positive, it requires much more treatment to secure eradication of syphilis, and, as an example, I would quote again Moore and Kemp, who with the same amount of treatment (thirteen to forty injections of " 606 " with mercury) as had given them cures in thirteen out of fourteen sero-negative primary cases obtained in I03 sero-positive and early secondary cases only sixty-nine cures. The authors state their belief that, with full co-operation on the part of the patient, 80 to 95 per cent. of patients in this class can be cured. I have quoted the view of Stokes that 75 per cent. of radical cures can be expected in primary and early secondary cases. Silberstein's results in sero-positive primary and early secondary were as follows; in private cases thirty-one primary, one serological relapse ; observation period in cases which did not relapse five to eight years, six cases; two to three and a half years, nine cases ; one to two years, seven cases ; half to one year, seven cases ; less than six months, one case. Early secondary (infection of two to six months' duration), thirty-one cases treated with a total of 0.8 to I.I salvarsan per series, six relapses ; forty-two treated with a total per series of 0.5 to 0.7 grm. salvarsan, twelve relapses, showing the importance of a sufficient dosage of salvarsan. The fifty-five patients who remained negative were observed after treatment as follows : five to eleven years, seven cases ; three to five years, twentythree cases ; one to two years, thirteen cases; a quarter to one year, twelve cases. The relapses occurred within six months in eleven cases. 


\section{BRITISH JOURNAL OF VENEREAL DISEASES}

In clinic cases, ten sero-positive primary cases gave two relapses; twenty-seven early secondary cases treated with the larger total dose per series, gave four relapses; and twenty-six treated with the smaller dose, six relapses. One of the relapses was detected after nine years, but he had been tested only once before this, viz., at the end of three months.

Altogether in sero-positive primary and early secondary the private cases showed $20 \cdot 2$ per cent. of relapses, and the clinic cases $22 \cdot 2$ per cent.

I can report at present only on my private cases, in which I have not yet had a relapse, clinical, serological or in the spinal fluid in secondary cases treated with thirty injections of an arsenobenzol compound (usually sulfarsenol subcutaneously) and as many of bismuth or mercury. Personally, I think it fairly safe to expect almost as high a proportion of success in early secondary cases (i.e., with infections of less than six months' duration) as in primary, provided that at least 50 per cent. more treatment is given.

I should like to discuss for a moment the difference between these results with well-conducted modern treatment and that of the pre-salvarsan era. It is sometimes said that mercury is, after all, the only true specific for syphilis and that arsenobenzol is only a symptomatic remedy. Yet I have always found that those who hold this view treat with arsenobenzol until the serum reactions are negative and resort to arsenobenzol again when they return to positive. Their views are based mainly on pre-Wassermann methods of judging results, and we know that the Wassermann test can put a totally different complexion on a case which seems to be cured. It is easy to believe one has obtained a higher percentage of cures if one does not test either blood serum or spinal fluid, seeing that the percentage of syphilitics who develop G.P.I. or tabes is not high, and large numbers of the other failures are camouflaged under death certificates of syncope, myocarditis, aortic incompetence, apoplexy, hepatic cirrhosis and so forth. Personally, I think that very few people have been cured with mercury. Certainly I found in some hundreds of soldiers whose blood I examined only three months after completion of two years' mercurial treatment that a very high proportion were not cured. No less than 40 per cent. were positive 


\section{PROGNOSIS OF SYPHILIS}

to the original Wassermann test, and over 70 per cent. to a modification which, whilst being wonderfully reliable, is more delicate than the method of the original test which I practised then. Scholtz and Willmer (Arch. $f$. Derm. $u$ Syphil., vol. I32) found 63 per cent. of relapses in 228 patients who had undergone five courses of mercurial treatment. I have already mentioned the results obtained by Glynn, Roberts and Bigland, which agree with Scholtz's and mine in showing that mercury is far behind arsenobenzol as a sterilising agent in syphilis. In saying this I am far from meaning that mercury has no place in the modern treatment of syphilis. I believe that it enhances the immediate effect of arsenobenzol, and this view is supported by the experiments of LehnhoffWyld (Annal de Malad. Vener., August, I924, and November, I926), who showed in animals that an arsenobenzol compound has a greater effect when there is present in the circulation another metal. But perhaps a greater value of combining arsenobenzol with mercurial or bismuthic treatment is in the prevention of neurorecurrence of the meningo-vascular type-cranial nerve palsies, paraplegia, hemiplegia and the like.

It seems convenient to discuss the incidence of this type of relapse under modern treatment before speaking of the rôle of mercury or bismuth in preventing it. A question of some importance is whether or not the patient who relapses after modern treatment, as I have defined it, is a worse case than if he had been treated by mercury alone. It has been stated by various clinicians that modern treatment tends to increase neuro-syphilis. I have seen no statistics bearing out this statement, and investigations which I initiated make me think that the statement is not well-founded. It is true that in the early days of salvarsan therapy there was a great increase in neurosyphilis, particularly of cranial nerve palsies, but this was very largely stopped by increasing the amount of treatment, and especially, as I hope to show, by combining salvarsan with mercurial treatment. The statement that neuro-recurrences have become more frequent has been repeated so much that I determined to make as full an investigation as I could into its truth, and some time ago I suggested the issue of a questionnaire by the Medical Society for the Study of Venereal Diseases to all venereal diseases treatment centres, to ask them how many men 


\section{BRITISH JOURNAL OF VENEREAL DISEASES}

who had been treated for syphilis in military hospitals during the war had reported at the treatment centres with neuro-recurrences. Answers were received from centres dealing with one-sixth the number of syphilitic patients treated in the public centres in England. The result disclosed eight cases, of which only three had received more than seven injections, and none had received more than twelve. The results were published in the British Journal of Venereal Diseases for April, I925, and in the same number all practitioners who had seen cases of syphilitic neuro-recurrence in ex-soldiers were invited to send in particulars. None further were forthcoming. With the aid of a grant from the Ministry of Health, and with the co-operation of the Medical Director-General, Ministry of Pensions, and of Dr. Barham andDr. Porter Phillips, a special investigation was made by $\mathrm{Mr}$. H. Nicol into the records of the Ministry of Pensions and those at Claybury and the Bethlem Royal Hospital. The results were published in the BRITISH JOURNAL OF VENEREAL DISEASES for July, I925, and showed only four certain and one possible case in which any arsenobenzol had been given before the onset of nerve symptoms, and in none of these had the number of injections exceeded ten. Considering that approximately I00,000 soldiers were treated with syphilis during the war, it does not seem as if the arsenobenzol with which they were treated had increased the incidence or hastened the onset of syphilitic neuro-recurrence. As I have already hinted, I believe this remarkable scarcity of ex-service men showing clinical signs of syphilitic neurorecurrence is due to the fact that in the treatment of their syphilis we gave mercury simultaneously with arsenobenzol. In practically all the early neuro-recurrences I have seen (e.g., paralysis of cranial nerves, most of them in the early salvarsan days), the patient had been treated only with arsenobenzol, and this has been one of my reasons for giving either mercury or bismuth at the same time. It would be beyond the scope of this paper to discuss reasons for the protective action of mercury or bismuth, if, indeed, I have not digressed enough already, but I should like to quote some figures which, I think, confirm my ideas on this subject.

In the exhaustive paper by Moore and Kemp from which I have already quoted, out of 402 cases of early 


\section{PROGNOSIS OF SYPHILIS}

syphilis, no less than fifty-eight are reported to have developed neuro-recurrences, and it is said that the " types of neuro-recurrence observed include acute syphilitic meningitis, cranial nerve palsies, the seventh and eighth being most frequently involved, precocious vascular neuro-syphilis, etc." The majority of the cases (55 per cent.) are said to have received six or less doses of arsenobenzol with no mercury. The authors' table shows that thirty-eight were in their Group I., that is, cases which had received one to eight injections of arsenobenzol, and in most of them " mercury was used either not at all or for less than one month." In contrast with this experience, I cannot remember to have seen one instance of neuro-recurrence after our treatment at the St. Thomas's Hospital, where we have dealt with approximately 7,000 cases of syphilis (of which, very approximately, 2,500 were early cases) since I920. Further, I am in a good position for receiving accounts of the experiences of medical officers of treatment centres throughout the country; none employing the simultaneous arsenobenzol-mercury method has told me of such a neuro-recurrence. In Silberstein's statistics are 3I7 cases of syphilis with infections of less than three years' duration. The records show only one neuro-recurrence of any kind, and fifty-one of the cases had been under observation for five or more years. The explanation which I offer is that in the Johns Hopkins Hospital Syphilis Clinic mercury and arsenobenzol are not given simultaneously ; the patient is first treated with "606," then has a course of mercurial inunctions, then more " 606 " and so forth, following their plan of continuous treatment. Under this form of treatment the spirochætes in the portions of the central nervous system to which the arsenobenzol is apparently unable to penetrate are unchecked in their action by either mercury or by any immune substance which would have developed if the syphilitic process had been allowed to simmer on throughout the body under purely mercurial treatment. We, on the other hand, give a course of arsenobenzol with mercury or bismuth, then a rest from injections, then another course, and so forth. Some would call our plan chronic intermittent treatment, but it is really continuous, as the mercury or bismuth is being absorbed throughout the interval. Scholtz, similarly, starts very early to give mercury. 


\section{BRITISH JOURNAL OF VENEREAL DISEASES}

To sum up the prognosis in primary and early secondary syphilis, I would say that modern treatment can effect . eradication in a very high proportion of cases. I have suggested earlier that the proportion of cures can be in the neighbourhood of Ioo per cent. when treatment is commenced before the serum reactions become positive, and not much below this figure when it commences between the arrival of the serum reactions and the sixth month of infection. This view is held also by Moore and Kemp, while Stokes takes a safer stand at 75 per cent. An adviser on assurance risks would, I take it, prefer to work on the lower figure, and assuming that a proposer declared a syphilitic infection and produced evidence of thorough treatment, as I have defined it, but had only recently finished treatment, I should say that he stood at least a three to one chance of being completely cured. Naturally the longer the period since completion of treatment, the greater the safety of assuming complete cure. Silberstein, as I have mentioned, considers that if a man has gone more than six months after treatment without a relapse, the chances are very strong that he will not do so in future, and Moore and Kemp consider, as a result of systematic spinal fluid examinations, that, if a patient shows no abnormality there after six months, he will not develop clinical neuro-syphilis or a positive fluid later unless a second opportunity of invasion occurs as manifested by a clinical or blood-serum recurrence. It would appear from this practically safe to say that in the case of a proposer who declared syphilis followed by thorough treatment and two years of observation during which he remained completely free from signs, clinical or serological, the history of syphilis could be ignored.

What of the relapse cases and those who at the time of the proposal show evidence of an infection of older standing than that with which I have so far dealt ? Are their chances of dying of syphilis less under modern methods of diagnosis and treatment than they were in the pre-Wassermann era, or not ? I think they are much less likely to die of syphilis, although I admit that the time is too early to base any judgment on the outcome of experience, and one's argument must be very much by inference:

The serum tests tell us that the disease has not been eradicated in cases where this fact was uncertain before 


\section{PROGNOSIS OF SYPHILIS}

the Wassermann test was discovered, and in this very large class of clinically latent syphilitics we can institute treatment long before active disease has damaged important organs. The question is whether or not such timely treatment protects these organs. For reasons which I will give, if we except the parenchyma of the brain, I think that it does. I admit that in many of these cases one cannot convert the serum and spinal fluid reactions to negative, though I do not think the absolutely pessimistic view taken by some workers on this point is justified considering that, by persevering for some years, I have quite often achieved the conversion. I ought to mention here that in cases with a persistently positive Wassermann reaction, Finger (Wien med. Woch., I926, January 2nd, p. 6), following Kyrle's (Wien Klin:Woch., I924, October 23rd) success in treating secondary syphilis by a course of malarial fever between two courses of "9I4," advocates the employment of malarial inoculation in all cases of syphilis of more than four years' standing. He states that, in such cases, the blood and spinal fluid promptly become negative, though sometimes only after three or four months. The method does not seem to have been practised in this country, and appears to deserve more attention. I will discuss the case, however, on the assumption that, at this later stage, we cannot secure complete eradication, and will take first the question of preventing cardio-vascular disease, as it is probably more expensive for assurance companies than any other form of syphilis. I judge that modern treatment can prevent cardio-vascular syphilis from the results we obtain in cases where there is evidence of active disease in the heart and great vessels. My own results confirm those of other workers in showing that, with careful and sufficiently prolonged treatment, patients with myocardial disease of a degree which is sufficient to interfere seriously with their work can be restored to a state of comparative robustness. I will cite two cases in my own practice. The first was a surgeon who had to give up practice on account of precordial distress on exertion and attack of angina. The diagnosis of myocarditis had been established by a cardiac specialist, and his serum reactions were strongly positive for syphilis. I treated him with sulfarsenol subcutaneously from April to June and from August to November, I92I. I was 


\section{BRITISH JOURNAL OF VENEREAL DISEASES}

unable to exceed a dose of 0.36 as higher doses provoked myocardial distress, but at the end of seven months he decided to return to work, and has now been following his old occupation strenuously for over five years. $\mathrm{He}$ had twenty-four doses of 0.30 sulfarsenol in I922, twenty in I923, and ten in I924. I saw him in February, I925, when he was in wonderful general condition, though he had a well-marked systolic and diastolic bruit in the aortic area and a systolic at the apex, with a fair amount of left-sided hypertrophy. His Wassermann reaction, which was strongly positive in June, I92I, and doubtful in November, I92I, was practically negative in February, I925, but the Kahn reaction was positive, and the Sigma test gave $3 \cdot 2$ units.

The second case came to me with a history of angina provoked by the least exposure of the skin to chill, with attacks of precordial heaviness and dyspnœa. His heart beat was irregular and the signs were those of myocarditis. He was treated on the same lines as the first case, but with bismuth also, and twelve months later was able to undergo the exertion of a day's shooting which involved his leaving home at 5 a.m. and returning at II p.m. Now, two years after commencing treatment, he can see no reason for continuing as he can do a day's work, play tennis, and, in a word, feels as well as ever. His blood reactions, which were strongly positive in January, I925, remained so until March, I926, but in September, I926, were practically negative.

In the same connection I would quote the results obtained by T. F. Cotton (B.M.J., May 22nd, I926, p. 855), who compared the progress of fifty-eight cases of cardio-aortic syphilis treated with regular courses of "9I4," mercury and iodide with that of fifty-eight control cases treated only symptomatically. At the time of writing fourteen, or $24 \cdot \mathrm{I}$ per cent., of the specifically treated cases had died at an average age of forty-five, while nineteen, or 33.9 per cent., of the control cases had died at an average age of forty-three. Stokes (Modern Clinical Syphilology, I926, W. B. Saunders Coy.), though cautious in his prognosis in the presence of active disease of the cardio-vascular system, indicates fairly plainly (p. 850) his view that, if one can start treatment of cardiovascular syphilis early enough, the outlook is satisfactory.

If one can obtain results such as I have quoted in 


\section{PROGNOSIS OF SYPHILIS}

cardio-vascular syphilis which is relatively far advanced, it seems justifiable to believe that in potential cases the heart and great vessels can be protected by modern treatment, and the percentage of casualties amongst the cases in which early treatment has failed to eradicate the disease should be very small. Everyone will agree that this is highly important from the point of view of prognosis of syphilis generally. Syphilis has gained its reputation as a great killing disease very largely through its effect on the cardio-vascular system. Moore and Kemp found in 266 cases of early syphilis which relapsed evidence of cardio-vascular disease in twenty-four, a substantial proportion considering the early stage at which the examinations were made. W. D. Smith (Boston Med. and Surg. Journ., I925, vol. I93, p. 387) quotes Lenz as saying that 25 per cent. of all syphilitics of the urban population of Germany die of aortitis or its consequences, as compared with 3 to 4 per cent. from general paresis, I to 2 per cent. from tabes, and Io per cent. from all other forms of visceral syphilis. Gürich (Münch. med. Woch., I925, June I2th, p. 980) in an analysis of 23,I79 autopsies in the General Hospital, Hamburg, found $806(3.4$ per cent.) with syphilitic disease of the organs, and of these $78 \cdot \mathrm{I}$ per cent. (women) to 86.5 per cent. (men) had aortitis, as contrasted with $I 5 \cdot 2$ per cent. with syphilis of the liver and I6.42 per cent. with syphilis of the central nervous system in the case of women and 3.7 per cent. liver and 23.5 per cent. central nervous system in men. The figures for syphilis of the central nervous system should have added to them something for general paresis, of which comparatively few would die in a general hospital. Lamb (quoted by Smith) estimates that 4 to 7 per cent. autopsies reveal syphilitic aortitis, and that 15 to 25 per cent. of organic heart disease is due to syphilis. This figure agrees fairly closely with that of Wittgenstein (Münch. med. Woch., I924, p. I354, ref. MacIlwaine and Campbell, Med. Ann., I926, p. 457), who found in 40,553 cases in the Berlin University, Poliklinik, that $8 \cdot 05$ per cent. had been treated for heart and visceral disease, and estimated that one-fifth of these were of syphilitic origin. He seems, however, to have estimated that the incidence of syphilitic disease of the central nervous system was the same as that of syphilis of the heart and viscera. This does not 


\section{BRITISH JOURNAL OF VENEREAL DISEASES}

agree with our mortality figures if one may estimate 75 per cent. of aortic insufficiency as due to syphilis, and 95 per cent. of aneurism, since the Registrar-General's mortality returns for I925 gave the following :-

$\begin{array}{clrr}\text { Deaths from } & \text { General paresis } & . & \text { I,539 } \\ \text {, } & \text { "Tabes . } & \text { T3I } \\ \text { " } & \text { " Aneurism } & . & \text { I,065 } \\ \text {, } & \text { ", Aortic valve disease. } & 3,079\end{array}$

Of course, there are other syphilitic diseases of the central nervous system which kill, but there are also very many of myocarditis (probably many more).

Warthin found 78 per cent. of syphilitic corpses with aortitis and 88 per cent. with syphilitic heart disease.

I think I have said enough to show that, by modern treatment, a very substantial proportion of mortality from syphilis can be prevented on the score of cardiovascular disease.

I cannot speak precisely on the prevention of hepatic disease by modern treatment. Arsenobenzol is not good for the liver, and in a case of syphilitic hepatitis I prefer to rely mainly on mercury or bismuth. The response to treatment in early cases is, however, good, so that it seems reasonable to expect that good treatment instituted on the score of a positive serum reaction would be protective.

To the question whether a patient with uncured syphilis, but as yet no signs of disease of the central nervous system, can be saved from this calamity there is a mixed answer. The good response of cases with syphilis of the supporting structures-meningitis and vascular lesionsindicates that, in respect of avoiding these, the outlook is good. Moore (Johns Hopkins Bulletin, I922, July, p. 23I) found a higher proportion with a positive cerebro-spinal fluid $(35 \cdot 2$ per cent.) in cases with a persistently positive blood reaction than in those with a negative ( $5_{5} .6$ per cent.). He divides cases with positive fluid into three groups according to the severity of the changes and the response to treatment. In Group I. the few cases which remained long enough under treatment had minor abnormalities which disappeared under treatment. In Group II., with rather more severe changes, eight out of thirteen remained free from symptoms, and in those which developed symptoms these disappeared. In Group III. eight cases were long enough under treatment to judge 


\section{PROGNOSIS OF SYPHILIS}

its effects. All remained serologically positive in spite of most persistent, including intra-spinal, treatment, and two developed symptoms, one becoming paretic. Besides the eight mentioned above there were nine who did not persist with treatment. Two of these became paretic, and a third developed cerebro-spinal syphilis. Altogether, from this study one gathers that, of forty-nine early cases of syphilis with a positive blood reaction, seventeen, or approximately a third, had positive fluid, and that in slightly under a third of these treatment failed to convert the reaction to negative. Further, it appears that of intractable cases of this kind, approximately a quarter developed clinical signs of neuro-syphilis. The calculation seems to work out to the effect that about 3 per cent. of those with a persistently positive blood reaction will develop clinical neuro-syphilis.

It is difficult to judge generally the effect of modern treatment on tabes, and therefore the probability of preventing it. On the whole my results have been good in early cases, but rather poor in those which were more advanced. In the treatment of general paresis, arsenobenzol and mercurial or bismuth treatment has been very disappointing, and I have seen one case which had received an enormous amount of treatment immediately prior to the onset of symptoms. It seems safe for the present to reckon in respect of general paresis on the same incidence in uncured cases as in the pre-salvarsan days.

I have spoken so far mainly on the question of protecting syphilitics from its killing effects, and in doing so have indicated my view that, in the event of disease of the cardio-vascular system, the liver, the meningo-vascular portion of the central nervous system, and even in tabes, much can be done to bring about arrest and so prolong life. But I have shown that I do not think much can be effected in general paresis by arsenobenzol and mercurial or bismuth treatment. It is necessary to qualify this, however, by a reference to the treatment of parenchymatous neuro-syphilis with tryparsamide, and also that with malarial inoculation. On tryparsamide opinions are rather divided, some speaking enthusiastically, others only coldly. O'Leary and Becker (Med. Journal and Record, March 3rd, I926), of the Mayo Clinic, report on fifty cases of general paresis observed for three years. In ten of these they obtained complete reversal of the 


\section{BRITISH JOURNAL OF VENEREAL DISEASES}

spinal fluid changes to negative and of the blood in eight. In regard to this, however, they say that in seven cases made serologically quite negative there was no associated clinical improvement. Their table shows marked improvement in six of the fifty cases, and they conclude that "there is a certain small group of patients with early paresis who derive marked benefit from tryparsamide." From this and other experiments, including my own, I do not think it would be safe as yet to calculate on any definite prolongation of life in general paresis by the use of tryparsamide. The malarial treatment seems to be in a different category, and, although at this stage it is impossible to assess its value, there seems to be no doubt that this is sufficiently important to warrant mention in this paper. Perhaps the latest statistics are those collected by Driver, Gammel and Karnosk (Jour. A.M.A., November 27th, I926, p. I82I). These show the results reported by thirty-six observers in different parts of the world in 2,336 cases of general paresis. Full remissions are reported in 27.5 per cent. of cases and incomplete remissions (moderate improvement) in 26.5 per cent. At this stage it is, of course, impossible to assess the real value of these percentages, as the play is by no means yet ended, but as an example of the fact that the remission can be a long one may be mentioned that WagnerJauregg (Psychiatr. neurol. Wochen., I9I8-I9, vol. 20, pp. I32 and 25I), who originated the method, inoculated, in I9I 7 , nine general paretics, and obtained four complete remissions. Of the four, as Gerstmann reported in 1925 (Die Malaria behandlung der progressiven Paralyse; Vienna, Julius Springer), one died a year later of pneumonia and empyema, while the remaining three were actively at work nearly seven and a half years later. It seems justifiable to hope that in a high proportion of those in whom complete remissions have been reported the disease has been arrested. At any rate, there can be no doubt that in malarial inoculation we have the means of prolonging life in the unhappy event of our other measures having failed. It is, in fact, our last trump.

Conclusions. - I have attempted to show in this paper:-

(I) That by earliest possible treatment on well-conducted lines a syphilitic infection can be eradicated in almost all cases, and that therefore the expectation of life is diminished only slightly. 


\section{PROGNOSIS OF SYPHILIS}

(2) That in a stage a little later than this, but within six months of infection, one can quite safely expect eradication in 75 per cent. of cases.

(3) That these results contrast with those obtained in the pre-salvarsan era, when cure of syphilis in the sense of eradication was probably effected in only a negligible proportion of cases.

(4) That in cases of older standing it is reasonable to expect that, by timely treatment, a very considerable proportion of disease of vital organs for which syphilis has gained its reputation as a killing disease of a very high order can be prevented.

(5) That the outlook in the worst event, the onset of general paresis, has been improved considerably by malarial treatment.

By inference from these conclusions it is clear that, in assessing the risk from a syphilitic infection, the medical adviser must consult the record as to treatment and serological examination, and must know the present state of the blood serum, if not also that of the spinal fluid. With this information in his possession and a report on the state of the vital organs, as judged by a very careful clinical examination, it should be possible to assess the risk with greater fairness to the candidate or the company than was ever possible before the advent of our modern methods. 